Two mechani sms for ming a combl i ke step pattern i nduced by a noving I i near adat om source

\begin{tabular}{|l|l|}
\hline 著者 & Sat o Nasahi de, M ur a Hi t oshi, Uwaha Naki o \\
\hline $\begin{array}{l}\text { j our nal or } \\
\text { publ i cat i on t i t l e }\end{array}$ & PHYSI CAL REVI EW \\
\hline vol une & E95 \\
\hline page r ange & 032803-1- 032803- 7 \\
\hline year & 2017- 03- 01 \\
\hline URL & ht t p: //hdl . handl e. net /2297/47766 \\
\hline
\end{tabular}




\title{
Two mechanisms forming a comblike step pattern induced by a moving linear adatom source
}

\author{
Masahide Sato, ${ }^{1}$ Hitoshi Miura, ${ }^{2}$ and Makio Uwaha ${ }^{3,4}$ \\ ${ }^{1}$ Information Media Center, Kanazawa University, Kakuma-machi, Kanazawa 920-1192, Japan \\ ${ }^{2}$ Department of Information and Basic Science, Nagoya City University, Mizuho-cho, Mizuho-ku, Nagoya 467-8501, Japan \\ ${ }^{3}$ Center for General Education, Aichi Institute of Technology, 1247 Yachigusa, Yakusa-cho, Toyota 470-0392, Japan \\ ${ }^{4}$ Department of Physics, Nagoya University, Furo-cho, Chikusa-ku, Nagoya 464-8602, Japan
}

(Received 20 October 2016; published 17 March 2017)

\begin{abstract}
We carry out phase field simulations to study properties of the comblike step patterns induced by an adatom source. When an adatom source advances right in front of a step, step wandering is caused by the asymmetry of the surface diffusion field and small protrusions are formed. If the velocity of the source $V_{\mathrm{p}}$ is smaller than a critical value $V_{\mathrm{p}}^{\mathrm{c}}$, the protrusions follow the adatom source with coarsening of the step pattern, and a regular comblike pattern with finger-like protrusions is formed. With a sufficiently small $V_{\mathrm{p}}$, the gap of the supersaturation is large at the adatom source. Since the period of protrusions, $\Lambda$, decreases with increasing $V_{\mathrm{p}}$, the coarsening of step pattern is irrelevant for the protrusions to catch up with the adatom source. Near $V_{\mathrm{p}}^{\mathrm{c}}$, the gap of the supersaturation at the adatom source is small. Taking account of the increase in $\Lambda$ with increasing $V_{\mathrm{p}}$, the coarsening of the step pattern is essential for the protrusions to follow the adatom source.
\end{abstract}

DOI: 10.1103/PhysRevE.95.032803

\section{INTRODUCTION}

An asymmetry of the surface diffusion field causes instabilities of straight steps on crystal surfaces. When adatoms are incorporated to a step from the lower terrace more easily than from the upper terrace by the Ehrlich-Schwoebel (ES) effect [1-3], the advancing step is unstable and step wandering occurs [4-8]. The motion of the step is described by the Kuramoto-Sivashinsky (KS) equation $[9,10]$, and the unstable step shows a chaotic behavior. When evaporation of atoms is absent, an array of regular grooves, which is perpendicular to the step, is formed on a vicinal face by in-phase step wandering $[11,12]$. The drift of adatoms induced by the direct electric current also causes a similar pattern [13-19].

Hibino et al. observed a comblike step pattern with fingerlike protrusions on a $\mathrm{Si}(111)$ vicinal face during $\mathrm{Ga}$ deposition [20]. The pattern is quite different from step patterns in previous studies [5,6,9-19]. The cause of the instability is the reconstruction of surface structure. When the temperature is about $580^{\circ} \mathrm{C}$, the surface structure on the $\mathrm{Si}(111)$ face is a $7 \times 7$ structure. By the deposition of $\mathrm{Ga}$, the surface structure is first changed to a $\sqrt{3} \times \sqrt{3}$ structure. When more Ga atoms are deposited on the surface, the surface structure is transformed to a $6.3 \times 6.3$ structure near the steps, and extra $\mathrm{Si}$ atoms are released from the phase boundaries of the two structures. The phase boundary in the lower side of a step mainly supplies $\mathrm{Si}$ atoms to the step. Since the phase boundary advances right in front of the step and is straighter than the step, it is regarded as an advancing straight adatom source moving right in front of the step.

Previously, we studied the step pattern induced by a moving linear adatom source in front of a step [21-24]. Since the amount of adatoms released from the source is less than that of the atomic layer, the straight step cannot follow the adatom source. When step wandering occurs, incorporation of adatoms mainly occurs near the tips of protrusions, and the protrusions catch up with the adatom source. In Monte Carlo simulations of a lattice model [21,22], the single step shows a comblike pattern consisting of finger-like protrusions when the step moves in the direction of the smallest step stiffness. The number of protrusions seemingly decreases in competition for growth, resulting in coarsening of the pattern. When the tips of the survived protrusions catch up with the adatom source, the coarsening stops and the protrusions advance steadily with the adatom source. The period of protrusions, $\Lambda$, is proportional to $V_{\mathrm{p}}^{-1 / 2}$, where $V_{\mathrm{p}}$ is the velocity of the adatom source. The relation between $V_{\mathrm{p}}$ and $\Lambda$ qualitatively agrees with experiment [20].

To control the noise strength and the step anisotropy of the system, we also carried out phase field simulations [23,24]. When $V_{\mathrm{p}}$ is larger than a critical value $V_{\mathrm{p}}^{\mathrm{c}}$, the step grows slower than $V_{\mathrm{p}}$ and unstable patterns [25] are formed in our simulation [23]. If the step anisotropy is small, the step shows a seaweed pattern: splitting of the protrusion occurs repeatedly and the main branch and side branches are not distinguished well. With the large step anisotropy, the step shows a dendritic pattern: the side branches are formed on the main branch. On the other hand, when $V_{\mathrm{p}}$ is smaller than $V_{\mathrm{p}}^{\mathrm{c}}$, the step follows the adatom source and a stable comblike pattern is formed. $V_{\mathrm{p}}^{\mathrm{c}}$ decreases with decreasing the step anisotropy. Without the anisotropy, the step pattern always looks irregular even with a small $V_{\mathrm{p}}$ in our simulation, so that the step anisotropy is necessary to form a regular comblike pattern. If $V_{\mathrm{p}}$ is much smaller than $V_{\mathrm{p}}^{\mathrm{c}}, \Lambda$ obtained in the simulations is about the same as that in Monte Carlo simulations of the lattice model [21]. Since the step has a finite width in the phase field model, fine structures smaller than the step width cannot be formed. In the phase field model with $V_{\mathrm{p}}$ close to $V_{\mathrm{p}}^{c}$, the diffusion limited aggregation (DLA)-like structure observed in the lattice model [21] is not formed, and the comblike pattern, in which $\Lambda$ increases with increasing $V_{\mathrm{p}}$, is formed. Considering the difference in the dependence of $\Lambda$ on $V_{\mathrm{p}}$, we conjectured that the mechanism forming the comblike pattern near $V_{\mathrm{p}}^{\mathrm{c}}$ is different from that of small $V_{\mathrm{p}}$ [23]. However, we did not have enough evidence to confirm the conjecture. In particular, our claim that coarsening is not really necessary at low velocity needs to be confirmed. Also, an analysis of the diffusion field in both low and high 
velocities is required to prove that the slow adatom source stabilizes the pattern, which is related to the unstable analytic solution given by Brener et al. [26] for the dendritic growth in a channel. To validate the mechanisms of the formation of the comblike pattern that we conjectured, we should investigate the properties of the pattern in the two velocity regions.

In this paper, we carry out phase field simulations and compare the properties of the comblike pattern with two different $V_{\mathrm{p}}$ which show the same $\Lambda$. We find a lot of evidence that validates our conjecture. In Sec. II, we introduce our model. In Sec. III, we show results of simulations and explain the mechanism that determine the period of protrusions. We analyze the properties of comblike patterns in the two $V_{\mathrm{p}}$ in detail. In Sec. IV, we summarize the results.

\section{PHASE FIELD MODEL}

Using a phase field model, we consider the time evolution of a step with a linear adatom source moving steadily toward the $y$ direction. The $x$ axis is taken to be parallel to the linear adatom source. The adatom source in front of the step advances releasing adatoms of a constant density $u_{0}$. The surface diffusion of adatoms occurs behind the adatom source. A phase field variable $\phi(x, y)$, which represents the surface height, is introduced. $\phi$ changes from -1 to 1 , where $\phi \approx 1$ in the upper side terrace and $\phi \approx-1$ in the lower side terrace. The step is expressed as a thin interface at $\phi=0$. The time evolution of the phase field variable $\phi$ is determined by the free energy $\mathcal{F}$, which is given by

$$
\mathcal{F}=\int d x d y\left[\frac{1}{2}\{W(\theta)\}^{2}|\nabla \phi|^{2}+F(\phi, u)\right],
$$

where $W(\theta)$ is a parameter determining the interface width. When $W(\theta)$ is sufficiently small, the region with large $|\nabla \phi|$ is thin and the phase field model expresses the step well. In our simulation, we assume that $W(\theta)$ has the fourfold symmetry given by

$$
W(\theta)=W_{0}\left(1+\epsilon_{4} \cos 4 \theta\right),
$$

where $W_{0}$ and $\epsilon_{4}$ are positive constants. $\theta$ is the angle normal to the step and defined as $\theta=\tan ^{-1}\left(\partial_{y} \phi / \partial_{x} \phi\right)$, where $\partial_{x} \phi=$ $\partial \phi / \partial x$ and $\partial_{y} \phi=\partial \phi / \partial y . F(\phi, u)$ is expressed as

$$
F(\phi, u)=f(\phi)-\lambda u g(\phi) .
$$

The parameter $u$ is the dimensionless supersaturation defined by $u=\Omega\left(c(x, y)-c_{\mathrm{eq}}^{0}\right)$, where $\Omega$ is the atomic area, $c(x, y)$ is the adatom density, and $c_{\mathrm{eq}}^{0}$ is the equilibrium adatom density for a straight step. The parameter $\lambda$ is a positive constant coupling the phase field and the density field. $f(\phi)$ is a double-well potential, and $g(\phi)$ increases monotonically with increasing $\phi$. In our simulation, $f(\phi)$ and $g(\phi)$ are given by

$$
\begin{gathered}
f(\phi)=-\frac{\phi^{2}}{2}+\frac{\phi^{4}}{4}, \\
g(\phi)=\phi-\frac{2 \phi^{3}}{3}+\frac{\phi^{5}}{5} .
\end{gathered}
$$

$F(\phi, u)$ has two minima at $\phi= \pm 1$. The regions with the negative $\phi$ and positive $\phi$ correspond to the lower and upper side terraces, respectively. In our simulations, initially $\phi$ decreases from 1 to -1 monotonically with increasing $y$ coordinate. When $u>0$, the region with positive $\phi$ expands with time to decrease $\mathcal{F}$. Thin region with large $|\nabla \phi|$ moves toward the $y$ direction, which expresses an advancing step.

Since the surface height is not conserved during growth, the time evolution of $\phi$ is given by $[27,28]$

$$
\begin{aligned}
\tau(\theta) \frac{\partial \phi}{\partial t}= & -\frac{\delta \mathcal{F}}{\delta \phi} \\
= & \nabla \cdot\left[W(\theta)^{2} \nabla \phi\right]-\frac{\partial F(\phi, u)}{\partial \phi} \\
& +\frac{\partial}{\partial x}\left[|\nabla \phi|^{2} W(\theta) \frac{\partial W(\theta)}{\partial \phi_{x}}\right] \\
& +\frac{\partial}{\partial y}\left[|\nabla \phi|^{2} W(\theta) \frac{\partial W(\theta)}{\partial \phi_{y}}\right],
\end{aligned}
$$

where $\tau(\theta)$ represents the relaxation time. We solve Eq. (6) with the diffusion equation for $u$ given by

$$
\frac{\partial u}{\partial t}=D \nabla^{2} u-\frac{1}{2} \frac{\partial \phi}{\partial t}-\nabla \cdot \boldsymbol{q},
$$

where $D$ is the diffusion coefficient and the first term on the right hand side represents the surface diffusion of adatoms. The second term represents mass conservation during crystallization by the change in $\phi$. The last term represents the change in the adatom density due to thermal noise. Since evaporation and impingement of adatoms are neglected, we use a conserved current noise satisfying

$$
\left\langle q_{i}(r, t) q_{j}\left(r^{\prime}, t^{\prime}\right)\right\rangle=2 D F_{u} \delta_{i, j} \delta\left(\boldsymbol{r}-\boldsymbol{r}^{\prime}\right) \delta\left(t-t^{\prime}\right),
$$

where $q_{i}(\boldsymbol{r}, t)$ represents the $i$ component of the current $\boldsymbol{q}(\boldsymbol{r}, t)$. To reproduce the thermal equilibrium fluctuation, the noise strength $F_{u}$ should satisfy $F_{u}=\Omega^{2} c_{\mathrm{eq}}^{0}$ [29]. However, we intentionally change $F_{u}$ in our simulation to study the dependence of the step pattern on the nonthermal noise strength.

The kinetic coefficient $K(\theta)$ and the capillary length $\tilde{d}(\theta)$, which is related to the step stiffness $\tilde{\beta}(\theta)$ as $\tilde{d}_{0}(\theta)=$ $\Omega^{2} c_{\text {eq }}^{0} \tilde{\beta}(\theta) / k_{\mathrm{B}} T$, are two important parameters in the step flow model. There is a model in which kinetic effects such as the ES effect [1-3] are taken into account [30], but we neglect this effect and consider a simple case. In terms of the parameters of the phase field model, $K(\theta)$ and $\tilde{d}(\theta)$ are expressed as [28]

$$
\begin{gathered}
K(\theta)=\frac{\lambda W(\theta)}{a_{1} \tau(\theta)}\left[1-a_{2} \lambda \frac{W(\theta)^{2}}{D \tau(\theta)}\right]^{-1}, \\
\tilde{d}_{0}(\theta)=\frac{a_{1}}{\lambda}\left[W(\theta)+\frac{d^{2} W(\theta)}{d \theta^{2}}\right]=d_{0}\left(1-15 \epsilon_{4} \cos 4 \theta\right),
\end{gathered}
$$

where $d_{0}=a_{1} W_{0} / \lambda$ with $a_{1}=5 \sqrt{2} / 8$ and $a_{2}=0.6267$. $W_{0}$ and $d_{0}$ are set to be $W_{0}=3$ and $d_{0}=5 \times 10^{-2}$ in our simulations. We consider the step motion in the limit of fast kinetics $[K(\theta) \rightarrow \infty]$, so that $\tau(\theta)$ is given by

$$
\tau(\theta)=\frac{a_{2} \lambda}{D} W(\theta)^{2}=\frac{a_{2} \lambda W_{0}^{2}}{D}\left(1-\epsilon_{4} \cos 4 \theta\right)^{2} .
$$

We use a square grid to solve Eqs. (6) and (7) by an explicit method. The system size is $L_{x} \times L_{y}$ with the grid 
spacing $\Delta x=\Delta y=1$. Initially, $\phi$ jumps from 1 to -1 at $y=y_{0}$ : the initial step position is $y=y_{0}$. The dimensionless supersaturation $u$ is set to be 0 far in $y<y_{0}$ and 0.5 far in $y>y_{0}$. The periodic boundary condition is used in the $x$ direction. During the simulation, $\phi$ and $u$ are kept constant at $y=0$ as $\phi(x, 0)=1$ and $u(x, 0)=0$. Th initial position of the adatom source is $y_{\mathrm{p}}=y_{\mathrm{s}}+3$, which is in the immediate vicinity of the step. In our simulations, the time increment is $\Delta t=0.2$. When the time increment exceeds $1 / V_{\mathrm{p}}$, we move the straight source by $\Delta y=1$. During the simulation, $\phi$ and $u$ vary only within $0<y<y_{\mathrm{p}}$ and are kept constant for $y>y_{\mathrm{p}}$ as $\phi=-1$ and $u=0.5$. We can regard the advance of the adatom source as a release of new adatoms with $u_{0}=0.5$ and $\phi=-1$ : a new row of a grid with $u=0.5$ is added in the system when the adatom source is moved and the system size is kept fixed with a zero flux boundary condition for all other time steps.

\section{NUMERICAL SIMULATION}

In our previous studies [23,24], a comblike pattern was formed when $V_{\mathrm{p}}$ is smaller than a critical value $V_{\mathrm{p}}^{\mathrm{c}}$. Since the number of adatoms released from the adatom source is half of the full atomic layer, a straight step cannot keep up with the adatom source. Initially, the distance between the step and the adatom source increases with increasing time. The gradient of supersaturation is formed between the step and the adatom source. The asymmetry of the surface diffusion field causes step wandering, and small protrusions are formed by the Mullins-Sekerka instability. When a protrusion grows higher [31] than others by the fluctuation of $u$, it outgrows lower ones, and those nearby protrusions die out.

If $V_{\mathrm{p}}$ is larger than $V_{\mathrm{p}}^{\mathrm{c}}$, the growing protrusions cannot catch up with the adatom source. Splitting of the tips of protrusions occurs, and the step pattern becomes irregular [23,24]. If $V_{\mathrm{p}}$ is smaller than $V_{\mathrm{p}}^{\mathrm{c}}$, the protrusions catch up with the adatom source and a comblike pattern with regular finger-like protrusions is formed, as shown in Fig. 1. When $V_{\mathrm{p}}=1.7 \times 10^{-2}$ [Fig. 1(b)], the number of high protrusions is five, which is larger than that with $V_{\mathrm{p}}=4 \times 10^{-3}$ [Fig. 1(a)]. However, two of them is lower than the others. The lower protrusions are weeded out and three protrusions survive. With $V_{\mathrm{p}}=1.7 \times 10^{-2}$, the tips of protrusions seem to be rounder and the distance between the tips and the adatom source is longer than those with $V_{\mathrm{p}}=4 \times 10^{-3}$ [23].

Figure 2 shows the relation between the period of protrusions, $\Lambda$, and the source velocity $V_{\mathrm{p}}$. The wavelength of the most unstable mode, $\lambda_{\max }$, is given by $\lambda_{\max }=2 \pi \sqrt{3 D d_{0} / V_{\mathrm{p}}}$ from a linear stability analysis [20]. In the initial stage, the step is expected to wander with $\lambda_{\max }$, which is expressed by a dash-dotted line in Fig. 2. With increasing time, the amplitude of step fluctuations increases and short protrusions are formed. We carry out the Fourier series expansion of the step form and calculate the most dominant wave number. From the wave number, we estimate the period of protrusions in an early stage, $\lambda_{\text {ini }}$, which turns out to be slightly larger than $\lambda_{\max }$. When the tips of protrusions catch up with the adatom source, the coarsening is terminated and the comblike structure with period $\Lambda$ appears. For small $V_{\mathrm{p}}, \Lambda$ is proportional to $V_{\mathrm{p}}^{-1 / 2}$ as $\lambda_{\text {ini }}$. However, $\Lambda$ increases with $V_{\mathrm{p}}$ for $V_{\mathrm{p}}$ larger than $10^{-2}$.
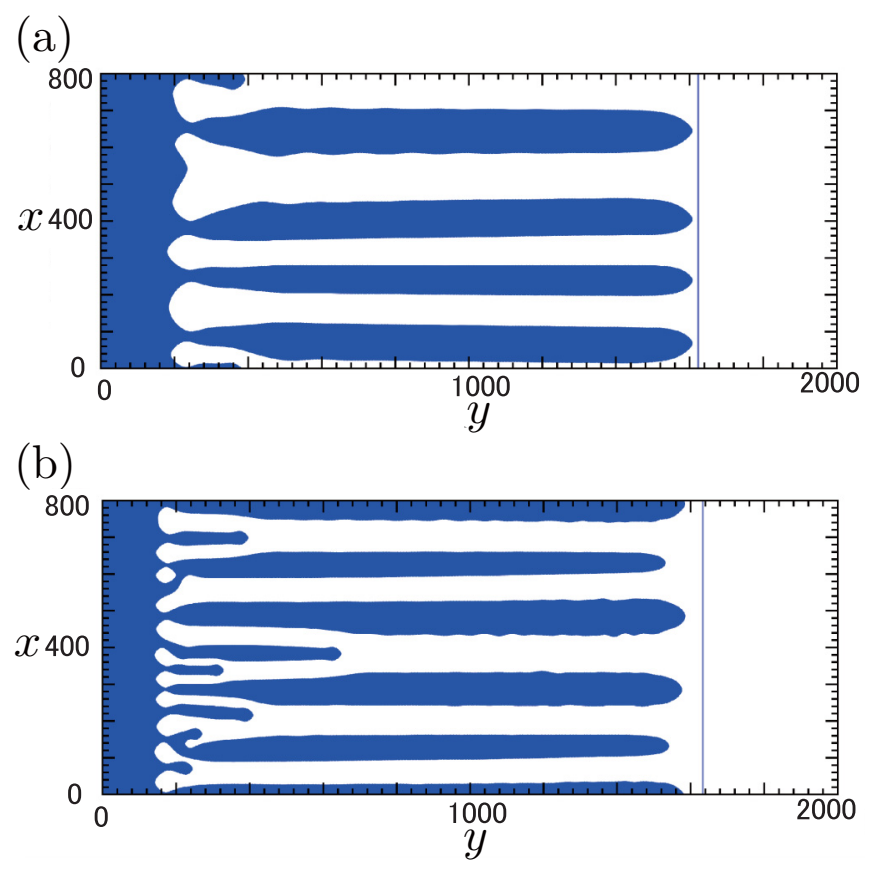

FIG. 1. Snapshots of the comblike pattern at (a) $t=3.8 \times 10^{5}$ and (b) $t=9 \times 10^{4}$. Parameters are $\epsilon_{4}=5 \times 10^{-2}, W_{0}=3, F_{u}=$ $10^{-5}$, (a) $V_{\mathrm{p}}=4 \times 10^{-3}$, and (b) $V_{\mathrm{p}}=1.7 \times 10^{-2}$. The region with $\phi>0$ is blue (dark) and the line parallel to the $x$ axis near the tips represents the position of adatom source.

Since the protrusions are periodic in the comblike structure, the supersaturation around a single finger-like protrusion in the comblike pattern with the period $\Lambda$ should be the same as the supersaturation around a finger-like protrusion growing in a channel with the channel width $\Lambda_{\text {channel }}$ Brener et al. [26] studied the steady growth of a needle-shaped crystal in a channel, in which the driving force is set to be constant at infinity. There are two branches of steady solutions: the fast

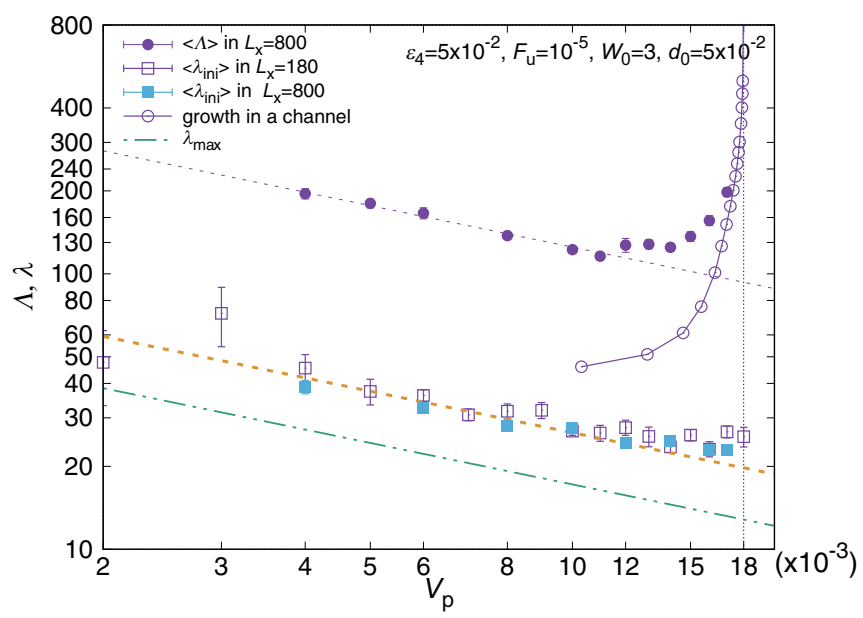

FIG. 2. Relation between the period of protrusions $(\Lambda$ or $\lambda)$ and the source velocity $V_{\mathrm{p}}$. The data are averaged over 10 or more runs. Relation between the channel width $\Lambda$ and the tip velocity $V_{\mathrm{p}}$ in dendritic growth in a channel is also shown. Dotted lines show $V_{\mathrm{p}}^{-1 / 2}$ fitting to the data. 
growing and slow growing solutions. In the branch of the slow growing solution, the steady velocity of the needle-shaped crystal, $V_{\text {needle }}$, increases with decreasing $\Lambda_{\text {channel }}$ as $\Lambda_{\text {channel }} \sim$ $V_{\text {needle }}^{-1 / 2}$, which is the same as the relation between $\Lambda$ and $V_{\mathrm{p}}$ in the small $V_{\mathrm{p}}$ case. In the branch of the fast growing solution, $V_{\text {needle }}$ increases with increasing $\Lambda_{\text {channel }}$. In our simulation, $\Lambda$ increases with increasing $V_{\mathrm{p}}$ near the critical velocity $V_{\mathrm{p}}^{c}$. The relation between $\Lambda$ and $V_{\mathrm{p}}$ (solid circles in Fig. 2) approaches that between $\Lambda_{\text {channel }}$ and $V_{\text {needle }}$ (open circles in Fig. 2). The relation between $\Lambda$ and $V_{\mathrm{p}}$ in our system is analogous to the relation between $\Lambda_{\text {channel }}$ and $V_{\text {needle }}$ in Ref. [26]. However, the stability of the solution is different. Pelcé [32] carried out a linear stability analysis and showed that the needle-shaped crystal in the slow growing branch in Ref. [26] is unstable, but in our simulation the comblike pattern is always stable.

In our previous paper [23], we suggested that the adatom source right in front of protrusions stabilizes the comblike pattern in the case of small $V_{\mathrm{p}}$. In the region, the scenario of the formation of the comblike pattern is as follows: The straight step is left behind the adatom source because the number of adatoms released from the adatom source is not enough for the step to follow the adatom source. The gradient of the supersaturation $u$ is formed in the diffusion field between the adatom source and the step. It causes the Mullins-Sekerka instability and small protrusions are formed. Since the adatoms supplied by the adatom source solidify around the tips of the protrusions, the protrusions grow fast and catch up with the adatom source. During the fast growth, the fluctuation of the height of the tips of protrusions causes the coarsening of the pattern because high protrusions get more adatoms and move faster than low protrusions. When surviving protrusions catch up with the adatom source, the fluctuation of the tip height is suppressed and the coarsening is terminated. In the case of small $V_{\mathrm{p}}$, the period of branches depends on the time interval for the tips of protrusions to reach the adatom source, and is influenced by the strength of the noise. In contrast, the period is determined by the steady solution in dendritic growth in a channel near $V_{\mathrm{p}}^{\text {(c) }}$, where the wider the period, the faster the protrusions grow.

The difference in the mechanism forming the comblike pattern affects properties of the comblike pattern. Hereafter, we carry out phase field simulations under various conditions and compare the properties in the two cases; $V_{\mathrm{p}}=4 \times 10^{-3}$ and $V_{\mathrm{p}}=1.7 \times 10^{-2}$, in which the average period of the protrusions is about the same, $\Lambda \approx 200$, as shown in Fig. 2 .

\section{A. Field of supersaturation}

In our previous paper [24], we showed that the tip velocity of the highest protrusion $V_{\mathrm{t}}$ converges to $V_{\mathrm{p}}$ after considerably exceeding $V_{\mathrm{p}}$ with a sufficiently small $V_{\mathrm{p}}$. The large excess of $V_{\mathrm{t}}$ over $V_{\mathrm{p}}$ does not occur with the velocity near $V_{\mathrm{p}}^{\mathrm{c}}$. The time evolution of $V_{\mathrm{t}}$ is related to the field of supersaturation $u$. Figure 3 shows the supersaturation $u$ and the step profile with the slow adatom source $\left(V_{\mathrm{p}}=0.004\right)$. In the early stage at $t=2.2 \times 10^{4}$ [Fig. 3(a)], the distance between the adatom source and the step increases. The gradient of supersaturation is formed between the step and the adatom source. The isoconcentration lines are parallel to the $x$ direction. The step fluctuates with a small amplitude. At $t=3.3 \times 10^{4}$ [Fig. 3(b)], (a)

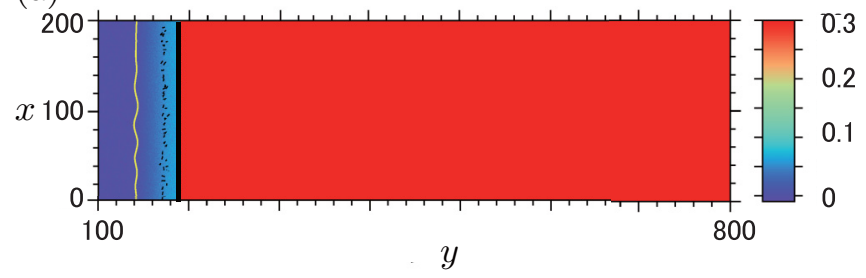

(b)

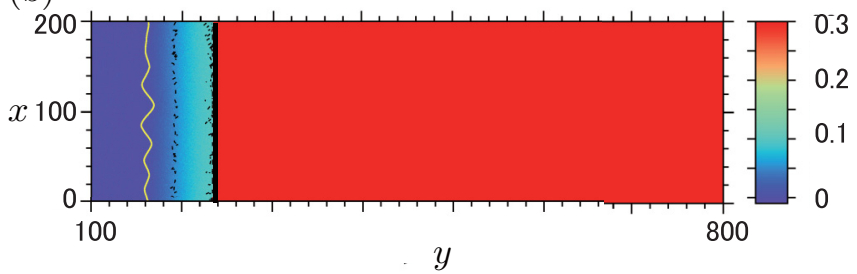

(c)

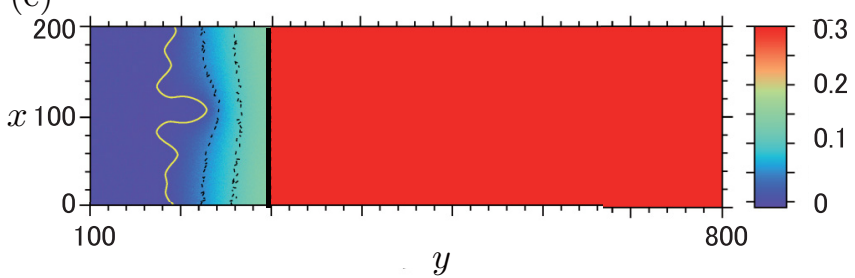

(d)

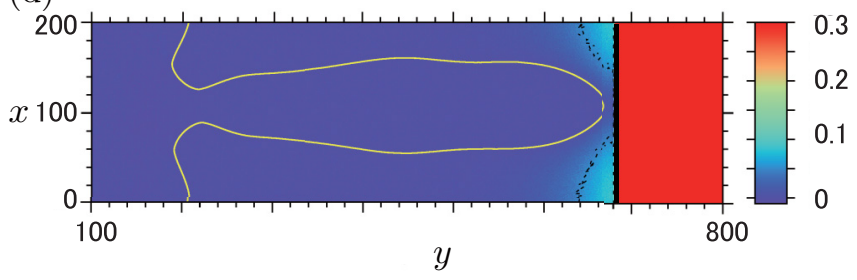

FIG. 3. Field of supersaturation and the step profile with the slow adatom source, $V_{\mathrm{p}}=4 \times 10^{-3} . \epsilon_{4}=5 \times 10^{-2}, W_{0}=3$, and $F_{u}=$ $10^{-5}$. Time is (a) $t=2.2 \times 10^{4}$, (b) $t=3.3 \times 10^{4}$, (c) $t=4.9 \times 10^{4}$, and (d) $t=1.45 \times 10^{5}$. Dotted lines represent isoconcentration lines separated by 0.05 . The thick solid line represents the position of the adatom source.

the amplitude of the fluctuation increases, and the MullinsSekerka instability occurs. In a later stage at $t=4.9 \times 10^{4}$ [Fig. 3(c)], short protrusions are formed. Since the high protrusion catches adatoms more than short protrusions, the lower protrusions lose the survival game and the number of protrusions decreases, resulting in coarsening of the step pattern. The isoconcentration lines are noticeably deformed near the tip of survived protrusion. In the late stage at $t=$ $1.45 \times 10^{5}$ [Fig. 3(d)], the deformed isoconcentration lines overlap with the adatom source, indicating a large jump of $u$ at the adatom source, and the gradient of supersaturation is small in front of the tip.

The adatom density in the growth near $V_{\mathrm{p}}^{\mathrm{c}}$ is different from that of the small $V_{\mathrm{p}}$. Figure 4 shows $u$ and the step profile with the fast adatom source $\left(V_{\mathrm{p}}=1.7 \times 10^{-2}\right)$. The number of protrusions formed in an early stage is twice more (see also Fig. 2) and the gradient of $u$ is much larger than that in Fig. 3(a). Otherwise, Figs. 3(a) and 3(b) are similar to Figs. 4(a) and 4(b), respectively. In Fig. 4(c), the gradient of $u$ in front of the tip of the protrusion is larger than that in Fig. 3(c). The 
(a)

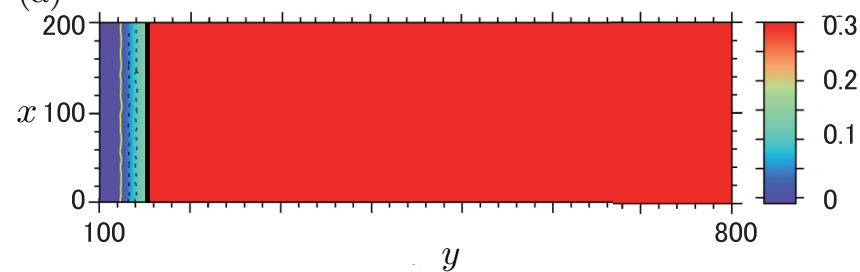

(b)

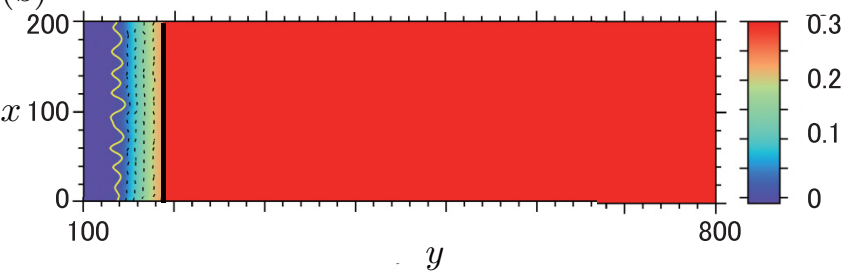

(c)

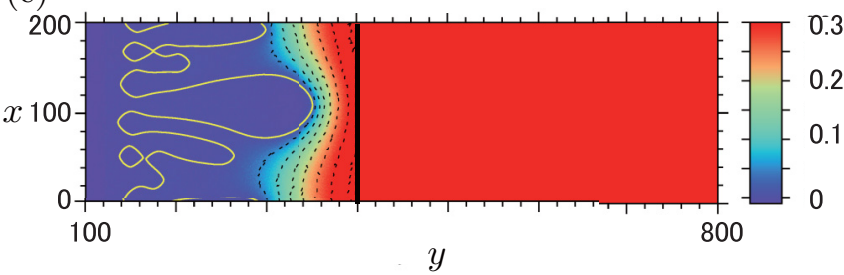

(d)

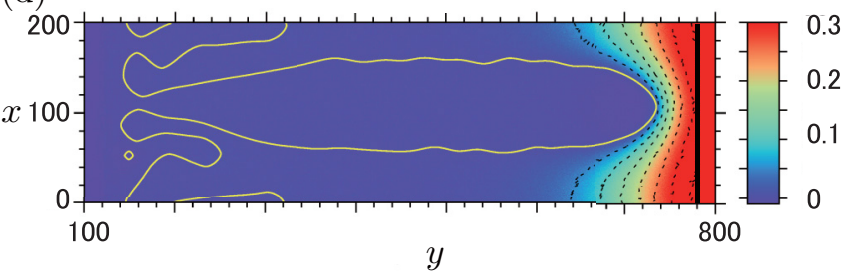

FIG. 4. Field of supersaturation $u$ and the step profile with the fast adatom source, $V_{\mathrm{p}}=1.7 \times 10^{-2} . \epsilon_{4}=5 \times 10^{-2}, W_{0}=3$, and $F_{u}=$ $10^{-5}$. Time is (a) $t=3 \times 10^{3}$, (b) $t=5 \times 10^{3}$, (c) $t=1.75 \times 10^{4}$, and (d) $t=4 \times 10^{4}$. Dotted lines represent isoconcentration lines of separated by 0.05 . The thick solid line represents the position of the adatom source.

shape of the highest protrusion is more rounded than that in Fig. 3(d). The field of supersaturation is very different in the last stage at $t=4.0 \times 10^{4}$ [Fig. 4(d)], and the jump of $u$ at the adatom source is much smaller than that in Fig. 3(d).

Figure 5 shows the dependence of the distance between the highest tip and the adatom source, $d_{\text {tip-source }}$, on $V_{\mathrm{p}} . d_{\text {tip-source }}$ hardly depends on $V_{\mathrm{p}}$ in the case of small $V_{\mathrm{p}}$, but $d_{\text {tip-source }}$ increases with increasing $V_{\mathrm{p}}$ near $V_{\mathrm{p}}^{\mathrm{c}}$, which also represents the difference in the field of supersaturation in the regions of small and large $V_{\mathrm{p}}$.

\section{B. Dependence of $\boldsymbol{\Lambda}$ on noise strength $\boldsymbol{F}_{\boldsymbol{u}}$}

Figure 6 shows the dependence of $\Lambda$ on the noise strength $F_{u}$ in the two $V_{\mathrm{p}}$ cases [33]. Previously, we studied the mechanism that determines $\Lambda$ in the case of small $V_{\mathrm{p}}$ [23]. $\Lambda$ depends on the time interval required for the tips of protrusions to catch up with the adatom source. It takes long time interval to form small protrusions with small $F_{u}$, so that a longer time is necessary for the exponentially growing protrusions to catch up with the adatom source. Thus, $\Lambda$ increases logarithmically

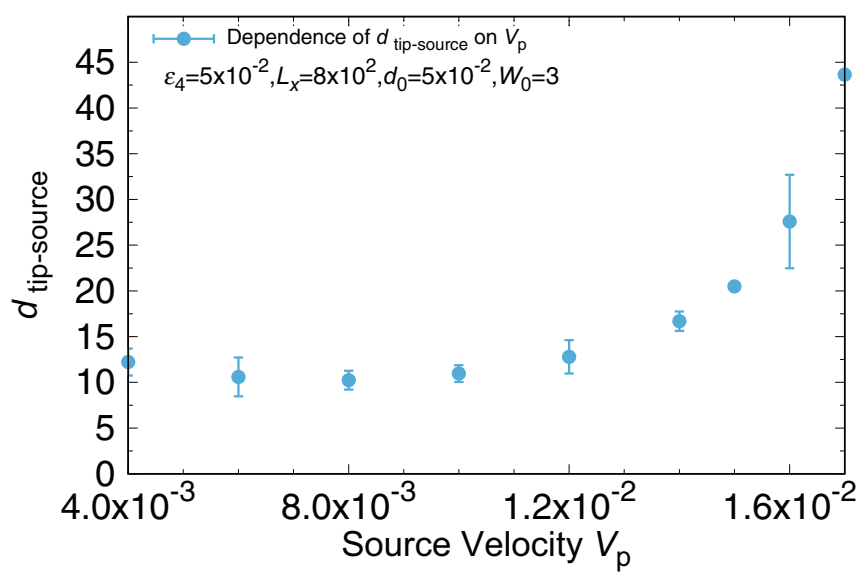

FIG. 5. Dependence of distance between the highest tip and the adatom source, $d_{\text {tip-source }}$, on $V_{\mathrm{p}}$. The parameters are $V_{\mathrm{p}}=1.7 \times 10^{-2}$, $\epsilon_{4}=5 \times 10^{-2}, W_{0}=3$, and $F_{u}=10^{-5}$. The system width is $L_{x}=$ 800 . The data are averaged over 10 runs.

with decreasing $F_{u}$ in the case of small $V_{\mathrm{p}}$ [23]. On the other hand, when $V_{\mathrm{p}}$ is near $V_{\mathrm{p}}^{\mathrm{c}}$, the relation between $\Lambda$ and $V_{\mathrm{p}}$ approaches that between the channel width and the steady growth velocity in dendritic growth in a channel. Since the steady tip velocity in dendritic growth in a channel should be independent of $F_{u}$, we expect that $\Lambda$ is not sensitive to $F_{u}$. In our simulations, the effect of $F_{u}$ on $\Lambda$ with large $V_{\mathrm{p}}$ is much weaker than that with small $V_{\mathrm{p}}$ as our expectation.

\section{Dependence of number of protrusions on system width}

The difference in the mechanism determining $\Lambda$ also affects the dependence of the number of protrusions $N$ on the system width $L_{x}$. We carry out 10 individual runs and count $N$ when the adatom source reaches to $L_{y}=1000$ or 2000 and the growth looks steady. Figure 7(a) shows $N$ with $V_{\mathrm{p}}=4 \times 10^{-3}$, which gives $\Lambda \approx 200$ and $\lambda_{\text {ini }} \approx 45$ (Fig. 2). When $100 \leqslant L_{x} \leqslant 220, N=1$ in all samples. Since $\lambda_{\text {ini }}$ is

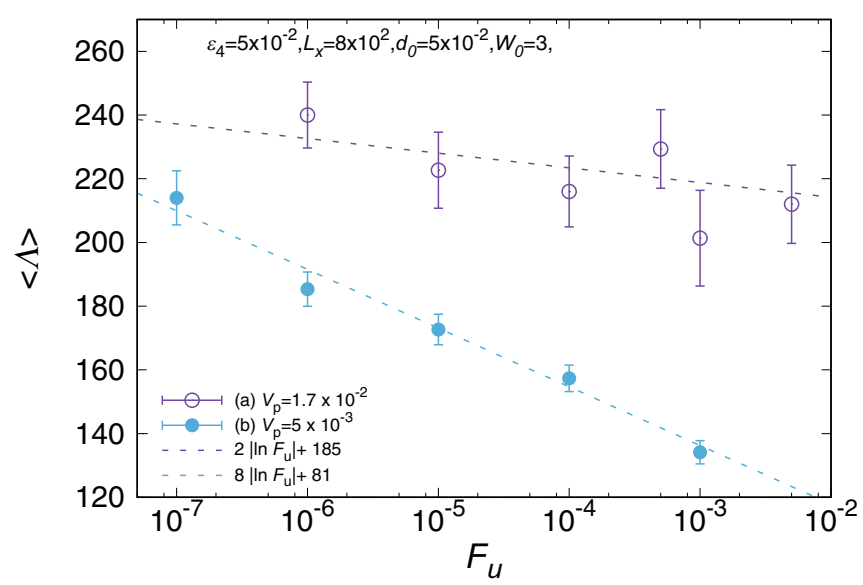

FIG. 6. Effect of the noise strength $F_{u}$ on the period $\Lambda$ with (a) $V_{\mathrm{p}}=1.7 \times 10^{-2}$ at $t=1.7 \times 10^{5}$ and (b) $V_{\mathrm{p}}=5 \times 10^{-3}$ at $t=$ $1.2 \times 10^{5}$. The system width is $L_{x}=800$. The tip position of the highest protrusion is about (a) 2900 and (b) 680. The data are averaged over (a) 10 runs and (b) 20 runs. 

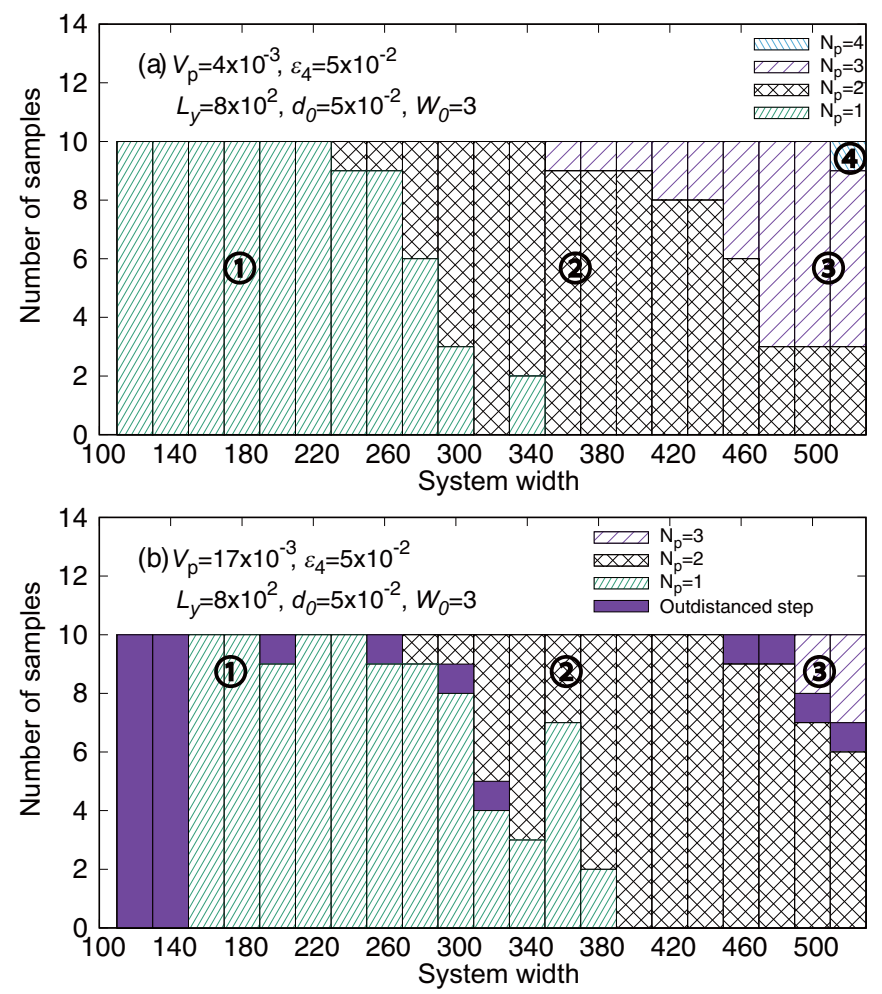

FIG. 7. Dependence of number of protrusions on $L_{x}$ with (a) $V_{\mathrm{p}}=4 \times 10^{-3}$ and (b) $V_{\mathrm{p}}=1.7 \times 10^{-2} \cdot F_{u}=10^{-5}$ and $L_{y}=1000$ or 2000 . Circled numbers represent the numbers of protrusions.

smaller than $L_{x}$, the Mullins-Sekerka instability occurs and the small protrusions are formed. When the coarsening proceeds up to the system size, further coarsening is prevented in the narrow system, but the survived protrusion catches up with the adatom source, which shows that the coarsening is not necessary to catch up with the adatom source. When $L_{x}$ is larger than 240, two protrusions are formed in samples. The number of samples with two protrusions increases with increasing $L_{x}$. The expected value of $N$ is one or two when $\Lambda<L_{x}<2 \Lambda$. However, three protrusions appear when $L_{x}=360$, which is smaller than $2 \Lambda$. In Fig. 2 , the period of protrusions decreases with increasing $V_{\mathrm{p}}$ in the case of the small $V_{\mathrm{p}}$, which means that protrusions with a small $\Lambda$ grow faster than those with a large $\Lambda$. Thus, the acceleration of protrusion by the coarsening of step pattern is not expected. If the regular array of the protrusions with a short period is accidentally formed, this array can catch up with the adatom source without coarsening. The similar relation between two protrusions and three protrusions is observed at $L_{x}=360$ and 380. Thus, Fig. 7(a) agrees with our expectation: the smaller the period is, the faster it grows as long as $\Lambda$ is much larger than $W_{0}$.

Figure 7(b) shows the dependence of $N$ on $L_{x}$ in the case of $V_{\mathrm{p}}=1.7 \times 10^{-2}$. One protrusion, which survived in competition with others, is outdistanced by the adatom source in all samples when $L_{x} \leqslant 140$. In the case of large $V_{\mathrm{p}}, \Lambda$ increases with increasing $V_{\mathrm{p}}$ in Fig. 2: a protrusions with large $\Lambda$ move faster than those with small $\Lambda$. Thus, the protrusions outdistanced by the adatom source shows that the acceleration
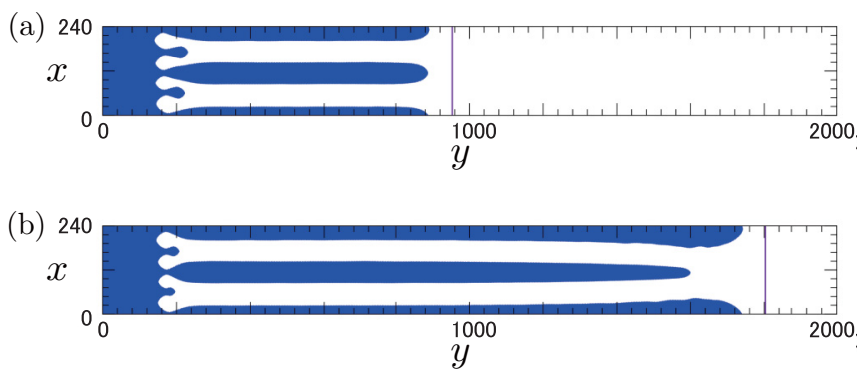

FIG. 8. Snapshots of the comblike pattern at (a) $t=5 \times 10^{4}$ and (b) $t=10^{5}$. Parameters are $\epsilon_{4}=5 \times 10^{-2}, W_{0}=3, F_{u}=10^{-5}, V_{\mathrm{p}}=$ $1.7 \times 10^{-2}$. The region with $\phi>0$ is blue (dark) and the line parallel to the $x$ axis near the tips represents the position of adatom source.

induced by the coarsening of step pattern is necessary for the protrusions to follow the adatom source.

Figure 8 shows snapshots of the comblike patterns in a sample with the system width $L_{x}=240$, and Fig. 9 shows the time evolution of $d_{\text {tip-source }}$. When $t=5 \times 10^{4}$, two protrusions with the same height survived [Fig. 8(a)], and the distance $d_{\text {tip-source }}$ increases with increasing time [Fig. 9]. Coarsening occurred and one protrusion remains at $t=10^{5}$ [Fig. 8(b)]. Since the protrusions interfere with each other via the surface diffusion field, the increase in captured adatoms per one protrusion by eliminating a competitor causes the acceleration of survived protrusions and $d_{\text {tip-source }}$ decreases (Fig. 9). In Fig. 7(b), protrusions not following the adatom source are also formed in some samples when $L_{x}>\Lambda$. In all cases, if we carry out simulations for very long time in a long system, the coarsening of the pattern will occur in a later stage and the survived protrusions catch up with the adatom source. From Figs. 8 and 9, it is reasonable to consider that the step outdistanced by the adatom source in Fig. 7 is only temporary. By reducing the number of protrusions it will eventually catch up with the adatom source.

\section{SUMMARY}

In this paper, we carried out the phase field simulations to study the properties of comblike step patterns in the

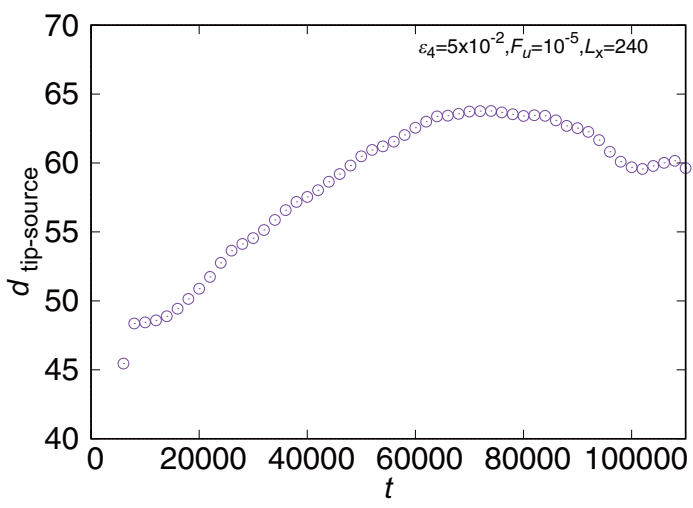

FIG. 9. Time evolution of $d_{\text {tip-source }}$. The parameters are $V_{\mathrm{p}}=$ $1.7 \times 10^{-2}, \epsilon_{4}=5 \times 10^{-2}, W_{0}=3$, and $F_{u}=10^{-5}$. The maximum system size is $L_{x} \times L_{y}=240 \times 1800$. 
two $V_{\mathrm{p}}$ cases with the same $\Lambda$. Owing to the difference in the mechanism determining $\Lambda$, the properties of a comblike pattern are different in the two cases although the period of the protrusions is the same. In the case of small $V_{\mathrm{p}}$, the velocity of the highest protrusion $V_{\mathrm{t}}$ once becomes much faster than that of the adatom source and decreases toward $V_{\mathrm{p}}$. The surface diffusion field is cut with the large gap of $u$ at the adatom source [Fig. 3(d)]. In Fig. 7(a), no protrusion is outdistanced by the adatom source in the narrow systems, which represents that the coarsening is not essential for the step to follow the adatom source. The steady pattern is a result of fluctuations and the exponential growth as inferred from Fig. 6 (see Ref. [23]). $\Lambda$ decreases with increasing $V_{\mathrm{p}}$ in Fig. 2, which indicates that protrusions are intrinsically unstable to splitting since it makes them grow faster. This feature is expected from the unstable branch of the analytic solution in the channel growth [26]. In the present situation with small $V_{\mathrm{p}}$, however, the protrusions are stabilized by the slowly moving adatom source since the supersaturation near the tips is very low [Fig. 3(d)].

In the case of large $V_{\mathrm{p}}$, the large overshoot of $V_{\mathrm{t}}$ seen in the case of small $V_{\mathrm{p}}$ does not occur. The gap of $u$ at the adatom source is much smaller [Fig. 4(d)]. Near $V_{\mathrm{p}}^{\mathrm{c}}$, the relation between $V_{\mathrm{p}}$ and $\Lambda$ approaches the relation between the steady growth velocity and the channel width in dendritic growth in a channel. In Fig. 7(b), protrusions cannot follow the adatom source when the system width is smaller than $\Lambda$ since $V_{\mathrm{t}}\left(=V_{\mathrm{p}}\right)$ is an increasing function of $\Lambda$. When the system size is larger than $\Lambda$, some protrusions are outdistanced by the adatom source. When the number of protrusions decreases by the coarsening, however, the survived protrusions accelerate and eventually catch up with the adatom source. These results indicate that the coarsening of step pattern is necessary for the protrusions to catch up with the adatom source in the case of large $V_{\mathrm{p}}$. The coarsening proceeds until the period $\Lambda$ becomes large enough for the protrusions to grow at the speed $V_{\mathrm{p}}$. Therefore, we expect that $\Lambda$ is not sensitive to the noise strength $F_{u}$ as found in Fig. 6.

\section{ACKNOWLEDGMENTS}

This work is supported by JSPS KAKENHI Grants No. JP26390054, No. JP16K05470, and No. JP15K05127.
[1] R. L. Schwoebel and E. J. Shipsey, J. Appl. Phys. 37, 3682 (1966).

[2] R. L. Schwoebel, J. Appl. Phys. 40, 614 (1969).

[3] G. Ehrlich and F. G. Hudda, J. Chem. Phys. 44, 1039 (1966).

[4] G. S. Bales and A. Zangwill, Phys. Rev. B 41, 5500 (1990).

[5] I. Bena, C. Misbah, and A. Valance, Phys. Rev. B 47, 7408 (1993).

[6] Y. Saito and M. Uwaha, Phys. Rev. B 49, 10677 (1994).

[7] T. Maroutian, L. Douillard, and H.-J. Ernst, Phys. Rev. Lett. 83, 4353 (1999).

[8] T. Maroutian, L. Douillard, and H.-J. Ernst, Phys. Rev. B 64, 165401 (2001).

[9] Y. Kuramoto and T. Tsuzuki, Prog. Theor. Phys. 55, 356 (1976).

[10] G. I. Sivashinsky, Acta Astronaut. 4, 1177 (1977).

[11] O. Pierre-Louis and C. Misbah, Phys. Rev. B 58, 2276 (1998).

[12] O. Pierre-Louis, C. Misbah, Y. Saito, J. Krug, and P. Politi, Phys. Rev. Lett. 80, 4221 (1998).

[13] M. Sato and M. Uwaha, Phys. Rev. E 60, 7120 (1999).

[14] M. Degawa, H. Nishimura, Y. Tanishiro, H. Minoda, and K. Yagi, Jpn. J. Appl. Phys. 38, L308 (1999).

[15] M. Degawa, H. Minoda, Y. Tanishiro, and K. Yagi, Surf. Sci. 461, L528 (2000).

[16] J.-F. Nielsen, M. S. Pettersen, and J. P. Pelz, Surf. Sci. 480, 84 (2001).

[17] M. Sato, M. Uwaha, and Y. Saito, Phys. Rev. B 62, 8452 (2000).
[18] M. Sato, M. Uwaha, Y. Saito, and Y. Hirose, Phys. Rev. B 65, 245427 (2002).

[19] M. Sato and M. Uwaha, J. Phys. Soc. Jpn. 65, 2146 (1996).

[20] H. Hibino, H. Kageshima, and M. Uwaha, Surf. Sci. 602, 2421 (2008).

[21] S. Kondo, M. Sato, M. Uwaha, and H. Hibino, Phys. Rev. B 84, 045420 (2011).

[22] S. Kondo, M. Kawaguchi, M. Sato, and M. Uwaha, J. Cryst. Growth 362, 6 (2013).

[23] M. Kawaguchi, H. Miura, K. Kishi, M. Sato, and M. Uwaha, Phys. Rev. E 91, 012409 (2015).

[24] K. Kishi, M. Kawaguchi, H. Miura, M. Sato, and M. Uwaha, e-J. Surf. Sci. Nanotechnol. 13, 269 (2015).

[25] E. Brener, H. Müller-Krumbhaar, and D. Temkin, Phys. Rev. E 54, 2714 (1996).

[26] E. Brener, M. Geilikman, and D. Temkin, Sov. Phys. JETP 67, 1002 (1988).

[27] A. Karma and M. Plapp, Phys. Rev. Lett. 81, 4444 (1998).

[28] A. Karma and W.-J. Rappel, Phys. Rev. E 57, 4323 (1998).

[29] A. Karma and W.-J. Rappel, Phys. Rev. E 60, 3614 (1999).

[30] G. Boussinot and E. A. Brener, Phys. Rev. E 88, 022406 (2013).

[31] We regard $y$ as a height variable.

[32] P. Pelcé, Europhys. Lett. 7, 453 (1988).

[33] In Fig. 6, the old data [23] with $V_{\mathrm{p}}=5 \times 10^{-3}$ are used for small $V_{\mathrm{p}}$ instead of $V_{\mathrm{p}}=4 \times 10^{-3}$ since the two velocities are very close. 